\title{
SEGAL ALGEBRAS ON NON-ABELIAN GROUPS
}

\author{
BY
}

\author{
ERNST KOTZMANN AND HARALD RINDLER
}

\begin{abstract}
Let $S^{1}(G)$ be a Segal algebra on a locally compact group. The central functions of $S^{1}(G)$ are dense in the center of $L^{1}(G)$. $S^{1}(G)$ has central approximate units iff $G \in[S I N]$. This is a generalization of a result of Reiter on the one hand and of Mosak on the other hand. The proofs depend on the structure theorems of [SIN]- and [IN]-groups. In the second part some new examples of Segal algebras are constructed. A locally compact group is discrete or Abelian iff every Segal algebra is rightinvariant. As opposed to the results, the proofs are not quite obvious.
\end{abstract}

Let $G$ be a locally compact group. $L^{1}(G)$ denotes the involutive Banach algebra of classes of complex-valued functions on $G$ integrable with respect to the Haar measure. (For reasons of practice we do not distinguish between the classes and the functions. We also write $f(x)=g(x)$ instead of $f(x)=g(x)$ for a.e. $x \in G$.) With $C^{0}(G)$, respectively $K(G)$, we denote the continuous functions vanishing at infinity, respectively, those with compact support. For the characteristic function of a subset $A \subseteq G$ we write $C_{A}$. For a closed subgroup $H$ of $G$ it is known that $G / H$ always has a quasi-invariant measure [7, Chapter 8, §1]. The corresponding $L^{1}$-space is denoted by $L^{1}(G / H)$. For $f \in L^{1}(G)$ we define

$$
T_{H, q} f(\dot{x})=\int_{H} \frac{f(x \xi)}{q(x \xi)} d \xi, \quad \pi_{H}(x)=\dot{x},
$$

where $\pi_{H}$ denotes the canonical morphism of $G$ onto $G / H$, and where $q$ is a strictly positive continuous function on $G$ satisfying

$$
q(x y)=q(x) \Delta_{H}(y) \Delta_{G}\left(y^{-1}\right), \quad x \in G, y \in H
$$

$\left(\Delta_{G}\left[\Delta_{H}\right]\right.$ denotes the Haar modules of $\left.G[H]\right) . T_{H, q}$ is a linear operator of $L^{1}(G)$ onto $L^{1}(G / H)$ with

$$
\left\|T_{H, q}\right\|_{1} \leqslant\|f\|_{1}, \quad f \in L^{1}(G) \text {, and } T_{H, q}(K(G))=K(G / H) \text {. }
$$

$G$ operates weakly continuously on $L^{1}(G)$ via the isometric operators $\Lambda_{y}, y \in$ $G$, defined by

Received by the editors November 15, 1976.

AMS (MOS) subject classifications (1970). Primary 22D05, 22D15, 43A15.

Key words and phrases. Locally compact group, group algebra, Segal algebra, center of an algebra, central function, approximate unit, compact invariant neighbourhood. 


$$
\Lambda_{y} \dot{f}(\dot{x})=\dot{f}\left(y^{-1} x\right) q\left(y^{-1} x\right) / q(x), \quad \dot{f} \in L^{1}(G / H) .
$$

We have

$$
T_{H, q} L_{y} f=\Lambda_{y} T_{H, q} f, \quad f \in L^{1}(G), y \in G,
$$

where $L_{y} f(x)=f\left(y^{-1} x\right)$. A detailed study of $T_{H, q}$ is given in [7, Chapter 3, $\S \S 1,2]$.

Let $S^{1}(G)$ always be a Segal algebra on $G$ in the sense of [8], i.e.

$\left(\mathrm{S}_{1}\right) S^{1}(G)$ is a dense linear subspace of $L^{1}(G)$.

$\left(\mathrm{S}_{2}\right) S^{1}(G)$ is a Banach space with respect to a norm \|\|$_{S}$ satisfying $\|f\|_{S}>\|f\|_{1}, f \in S^{1}(G)$.

$\left(\mathrm{S}_{3}\right) y \rightarrow L_{y}$ is a weakly continuous representation of $G$ by isometric operators on $S^{1}(G)$.

In particular, it follows that $S^{1}(G)$ is a left ideal in $M^{1}(G)$, the involutive Banach algebra of all bounded measures on $G$. A detailed study of Segal algebras is given in [8].

We briefly recall the classes of locally compact groups that we will need. $B$ is always a subgroup of $\operatorname{Aut}(G)$, the group of all continuous automorphisms, containing all inner automorphisms.

$[I N]_{B}$ : $G$ contains a compact, $B$-invariant neighbourhood of the identity.

$[S I N]_{B}: G$ contains a fundamental system of $B$-invariant neighbourhoods of the identity.

$[F I A]_{B}^{-}: B$ is precompact in $\operatorname{Aut}(G)$.

If $B$ is the subgroup of all inner automorphisms, we write [IN], [SIN], [FIA]. The reader can find a detailed discussion in [1]. In general, the notation of [7] is used in this paper.

Analogously to the paper [6] of Mosak we characterize the class [SIN] by the existence of central approximate units in a Segal algebra. In contrast to the case $L^{1}(G)$ it is not trivial that there must always exist central approximate units in any given Segal algebra. This result also complements partially that of Reiter, who considered special Segal algebras on compact groups [8, $\S 17$, Theorem 1].

THEOREM 1. The following statements are equivalent:

(a) $G \in[S I N]$.

(b) $S^{1}(G)$ has multiple approximate units contained in the center of $S^{1}(G)$.

REMARK. In the proof of $(a) \Rightarrow(b)$ we construct multiple approximate units of central functions which are even bounded in $L^{1}(G)$. Therefore, Theorem 1 of $[8, \S 9]$, formulated for right ideals, is true for every Segal algebra on a $[S I N]$-group.

ProOF. (b) $\Rightarrow$ (a) Let $\left(u_{\alpha}\right)_{\alpha \in A}$ be multiple approximate units of $S^{1}(G)$. For $x \neq e$, the operator $L_{x}$ cannot reduce to identity on $\left(u_{\alpha}\right)_{\alpha \in A}$ because $S^{1}(G)$ is 
dense in $L^{1}(G)$. We repeat the arguments of [6] to prove $G \in[S I N]$.

We write $Z^{1}(G)\left[Z^{s}(G)\right]$ for the center of $L^{1}(G)\left[S^{1}(G)\right]$.

(a) $\Rightarrow$ (b) From [1, (2.13) Theorem] we obtain that $G$ contains an open, normal subgroup $H=V \times K$, where $V$ is a vector group and $K$ is a compact group, such that $H \in[F I A]_{B}^{-}$( $B$ denotes the group of the inner automorphisms of $G$ restricted to $H$ ). Moreover, we may assume that both $V$ and $K$ are $B$-invariant [1, (1.1) Theorem]. For a finite subset $F \subset S^{1}(G)$ and $\varepsilon>0$, we can take $u \in Z^{1}(G)$, supp $u \subset H, u>0,\|u\|_{1}=1$, such that

$$
\|u * f-f\|_{s}<\varepsilon / 2, \quad f \in F,
$$

holds; this follows from $\left(\mathrm{S}_{3}\right)$ and [G]. If we can find a $v \in Z^{s}(G)$ with

$$
\|u-v\|_{1}<\varepsilon / 2 M, \quad M=\max \left\{\|f\|_{S} \mid f \in F\right\}, \quad\|v\|_{1}=1 .
$$

(1) and (2) yield

$$
\|v * f-f\|_{s}<\varepsilon, \quad f \in F .
$$

We consider $u$ as an element of $L^{1}(H)$. It follows from [11, Theorem 3] that we can approximate $u$ by functions of the form $w=\sum_{n=1}^{N} f_{n} \otimes \chi_{n}$, where $f_{n} \in L^{1}(V)$ with $\hat{f}_{n} \in K(\hat{V}), 1 \leqslant n<N$, and $\chi_{n}, 1 \leqslant n<N$, are characters of $K$ (see [7, Chapter 5, $\$ 4.1(1)]$ and [10, p. 85]). Since $B$ is precompact in Aut $H$, we can form

$$
w^{\#}=\int_{B} S_{\alpha} w d \alpha,
$$

where $S_{\alpha} w(x)=w(\alpha x), \alpha \in B^{-}$. Since $B^{-}$is compact and the Fourier transform of $w$ has compact support, we have $w^{\#}=\sum_{m=1}^{M} g_{m} \otimes \chi_{m}$, where $g_{m} \in L^{1}(V)$ with $\hat{g}_{m} \in K(\hat{V})$. For $e=\tau \otimes \sum_{m=1}^{M} \chi_{m}$ with $\hat{\tau} \in K(\hat{V})$ and $\hat{\tau}(\hat{V})=1, \hat{v} \in \cup_{1<m<M} \operatorname{supp} \hat{g}_{m}$, we have $e^{\#}{ }^{*} w^{\#}=w^{\#}$ (here the convolution is that of $L^{1}(G)$ ). Since $\left\|u-w^{\#}\right\|_{1}<\|u-w\|_{1}$ holds, the proof is finished by the following:

LEMMA 1. Let $S^{1}(G)$ be a Segal algebra on a locally compact group $G$, and let $F$ be a subset of $L^{1}(G)$. If there exists an element $e \in Z^{1}(G)$ with $f * e=f$, $f \in F$, then there is an element $e^{\prime} \in S^{1}(G)$ with $f * e^{\prime}=f, f \in F$, in particular, $F \subset S^{1}(G)$.

Proof. Take $g \in S^{1}(G)$ with $\|g-e\|_{1}<1$ and set

$$
e^{\prime}=\left(e+\sum_{n>1}(e-g)^{n}\right) * g
$$

(here $(e-g)^{n}$ denotes the $n$th convolution product of $e-g$ ). Setting $h=e+$ $\sum_{n>1}(e-g)^{n}$, we obtain

$$
f * h-f * h *(e-g)=f * h * g .
$$

On the other hand, the left side of (3) is equal to 


$$
\begin{gathered}
f *\left(e+\sum_{n>1}(e-g)^{n}-e *(e-g)-\sum_{n>2}(e-g)^{n}\right) \\
=f *(e+(e-g)-e *(e-g))=f .
\end{gathered}
$$

REMARK. We de not know a direct proof of Theorem 1 without using structure theorem of $[1,(2.13)]$.

Let $A$ be a closed subalgebra of $L^{1}(G)$; it is not trivial whether [ $A \cap$ $S^{1}(G) \Gamma=A$.

THEOREM 2. The center $Z^{s}(G)$ of every Segal algebra is dense in the center $Z^{1}(G)$ of $L^{1}(G)$.

REMARKS. (i) It follows from [6], that $G \in[I N]$ iff $Z^{s}(G) \neq\{0\}$ for every Segal algebra.

(ii) It is constructive to consider the theorem for the groups $S_{3} \times \mathbf{R}\left(S_{3}\right.$ is the symmetric group of order 6) and for the semidirect product $\mathbf{R} \times{ }_{s} \mathbf{Z}(2)$ $(\mathbf{Z}(2)$ denotes the cyclic group of order 2). Even for these simple cases the proof is not obvious. For example, let us discuss the second case $G=\mathbf{R}$ $\times{ }_{s} Z(2) . L^{1}(G)$ can be identified with $L^{1}(\mathbf{R}) \times L^{1}(\mathbf{R})$ as a Banach space. A simple computation shows that $f \in Z^{1}(G)$ iff $f=\left(f_{1}, 0\right), f_{1} \in L^{1}(G)$ with $f_{1}=f_{1}^{\vee}\left(f_{1}^{\vee}(x)=f_{1}\left(x^{-1}\right)\right)$. In general, it is not true that $\left(f_{1}, f_{2}\right) \in S^{1}(G)$ implies $\left(f_{1}, 0\right) \in S^{1}(G)$ (even for the direct product $\mathbf{Z}(2) \times \mathbf{R}$ we can take $S^{1}(G)=\left\{\left(f_{1}, f_{2}\right) \mid f_{1}, f_{2} \in L^{1}(G), \hat{f}_{1}+\hat{f}_{2} \in L^{1}(\mathbf{R})\right\}$ as a counterexample). Moreover, $\left(f_{1}, 0\right) \in S^{1}(G)$ does not imply $\left(f_{1}^{\vee}, 0\right) \in S^{1}(G)[3$, p. 181].

Proof. We can restrict our attention to the case $G \in[I N]$. Since $Z^{s}(G)=$ $Z^{1}(G) \cap S^{1}(G)$ holds, we may assume that $Z^{1}(G) \neq\{0\}$-otherwise there is nothing to prove. But then $G$ belongs to [IN] [6].

We may even assume that $G \in[S I N]$. If $G \in[I N]$, there is a compact normal subgroup $K$ such that $G / K \in[S I N]$ [1, (2.3) Theorem]. Moreover, the center $Z^{1}(G / K)$ of $L^{1}(G / K)$ is isometrically isomorphic to $Z^{1}(G)$ via $\dot{f} \rightarrow \dot{f} \circ \pi_{K}, \dot{f} \in L^{1}(G / K)$, where $\pi_{K}$ is the canonical homomorphism of $G$ on $G / K$. Since $f \in S^{1}(G)$ implies $m_{K} * f \in S^{1}(G)$, where $m_{K}$ is the Haar measure of $K$, and since $g \in Z^{1}(G)$ implies $m_{K} * g=\dot{g} \circ \pi_{K}$, we need only consider the case $G \in[S I N]$.

Let $f \in Z^{1}(G), \varepsilon>0$. We choose $g \in S^{1}(G)$ with

$$
\|f-g\|_{1}<\varepsilon \text {. }
$$

By Theorem 1 there exists $u \in Z^{S}(G),\|u\|_{1}<K$ ( $K$ is a independent constant; see Remark at Theorem 1) with

$$
\|g * u-g\|_{S}<\varepsilon
$$

Now, (4) and (5) imply that

$$
\|f * u-f\|_{1} \leqslant\|u\|_{1}\|f-g\|_{1}+\|g * u-g\|_{1}+\|f-g\|_{1} \leqslant(2+K) \varepsilon
$$

holds. 
Remarks. (i) It follows from [5, p. 149] that the center of $L^{1}(G)$ is isometrically isomorphic to the center of the group algebra of an $[F I A]_{B}$ group. We do not see any way to simplify the last proof using this fact, i.e. to prove the theorem without using Theorem (2.13) of [1]. However, the result of Theorem 2 is independent of group structure. It would be of interest if Theorem 2 is true for general dense Banach ideals.

(ii) Analogously to [4] we can prove: The following statements are equivalent:

(a) $G \in[F I A]$.

(b) For every Segal algebra $S^{1}(G)$ the closed two-sided ideal $S^{0}(G)=$ $\left\{f \mid f \in S^{1}(G), \int f=0\right\}$ has multiple approximate units belonging to the center of $S^{0}(G)$. The proof is the same as in [3], using Theorems 1 and 2.

If $G$ is a discrete or locally compact Abelian group, every Segal algebra is invariant under the right translation operators $R_{y}, y \in G$, where

$$
R_{y} f(x)=f\left(x y^{-1}\right) \Delta_{G}\left(y^{-1}\right), \quad g \in L^{1}(G) .
$$

(For discrete $G, L^{1}(G)$ is a Banach algebra with unit; therefore, there does not exist any dense left ideal except $L^{1}(G)$.) B. E. Johnson constructs non-right-invariant Segal algebras on very special groups in [3, p. 182]. Constructing some new examples of Segal algebras, especially such, which are not-right-invariant, by the way, we have the following:

THEOREM 3. The following statements are equivalent:

(a) $G$ is discrete or Abelian.

(b) Every Segal algebra is right invariant.

The proof $(a) \Rightarrow(b)$ is trivial. To prove $(b) \Rightarrow(a)$ we construct on every locally compact group, which is neither discrete nor Abelian, a non-rightinvariant Segal algebra.

Definition. Let $H$ be a closed (not necessarily normal) subgroup of $G$. A dense linear subspace $S^{1}(G / H)$ is called a Segal module, if the following conditions are fulfilled:

(i) $S^{1}(G / H)$ is a Banach space with respect ot a norm \|\|$_{\dot{S}}$ such that $\|\dot{f}\|_{1} \leqslant\|\dot{f}\|_{\dot{s}}, \dot{f} \in S^{1}(G / H)$.

(ii) $S^{1}(G / H)$ is invariant under $\bigwedge_{y}, y \in G$ and $y \rightarrow \bigwedge_{y} \dot{f}$ is a continuous function of $G$ in $S^{1}(G / H)$ for every $\dot{f} \in S^{1}(G / H)$.

(iii) $\left\|\wedge_{y} \dot{f}\right\|_{\dot{s}}=\|\dot{f}\|_{\dot{s}}, \dot{f} \in S^{1}(G / H)$.

EXAMPLES. (a) If $H$ is normal, $S^{1}(G / H)$ is a Segal algebra.

(b) If $G / H$ has an invariant measure, $L^{1} \cap L^{p}(G / H), 1<p<\infty$, and $L^{1} \cap C^{0}(G / H)$ are Segal modules with the norms $\|\dot{f}\|_{\dot{s}}=\|\dot{f}\|_{1}+\|\dot{f}\|_{p}$, respectively, $\|\dot{f}\|_{\dot{s}}=\|\dot{f}\|_{1}+\|\dot{f}\|_{\infty}$.

(c) We write $l^{1}$ for the Banach space of all complex-valued absolutely 
summable sequences $\mathbf{a}=\left(a_{n}\right)_{n>1}$ with the usual norm $\|\mathbf{a}\|_{1}=\Sigma\left|a_{n}\right|$. Let $\dot{\mathbf{g}} \neq 0$ be a positive function of $K(G / H)$. Consider the functions $\dot{f}$ on $G / H$ such that there exist $\mathrm{a} \in l^{1}$ and $y_{n} \in G, n \geqslant 1$, with

$$
|\dot{f}(\dot{x})|<\sum a_{n} \Lambda_{y_{n}} \dot{g}(\dot{x}), \quad \dot{x} \in G / H .
$$

Setting $\|\dot{f}\|_{W}=\inf \left\{\|\mathbf{a}\|_{1} \mid \mathbf{a} \in l^{1}\right.$ satisfying (6) $\}, f \in K(G / H)$, the completion $W^{1}(G / H)$ of $K(G / H)$ under the norm \|\|$_{W}$ forms a Segal module. ${ }^{1}$

Proposition 1. Let $S^{1}(G / H)$ be a Segal module. $T_{H, q}^{-1}\left(S^{1}(G / H)\right)$ is a Segal algebra on $G$ with the norm $\|f\|_{S}=\|f\|_{1}+\left\|T_{H, q} f\right\|_{S}, f \in T_{H, q}^{-1}\left(S^{1}(G / H)\right)$.

PROof. This is a straightforward calculation left to the reader.

REMARK. Let $G$ be the " $a x+b$ "-group, i.e. the semidirect product of $\mathbf{R}$ with $\mathbf{R}_{+}^{*}$, and $H=\mathbf{R}_{+}^{*}$ (one can also take the semidirect product of the $p$-adic numbers $\mathbf{Q}_{p}$ with $\mathrm{Z}$ and choose $H=\mathrm{Z}$ ). For every Segal algebra $S^{1}(G)$ we obtain $T_{H, q}\left(S^{1}(G)\right)=L^{1}(G / H)$; in particular, from Proposition 1 it follows that $L^{1}(G / H)$ is the only Segal module on $G / H$.

We have $G / H \cong \mathrm{R}$, and the relatively invariant measure on $G / H$ is the Lebesgue measure on $\mathbf{R}$. An easy calculation shows that

$$
\Lambda_{y} \dot{f}(\dot{x})=\alpha^{-1} f\left(-\alpha^{-1} a+\alpha^{-1} \dot{x}\right), \quad \dot{x} \in \mathbf{R}, y=(a, \alpha) \in G, \dot{f} \in L^{1}(R),
$$

holds. Therefore, $S^{1}(\mathbf{R})=T_{H, q}\left(S^{1}(G)\right)$ with the quotient norm is a Segal algebra, on which the operators $M_{\rho}, \rho>0\left(M_{\rho} \dot{f}(\dot{x})=\rho \dot{f}(\rho \dot{x}), \dot{x} \in \mathbf{R}, \dot{f} \in\right.$ $\left.L^{1}(\mathbf{R})\right)$ acts as isometries. For $\int \dot{g}=1, \dot{g} \in S^{1}(\mathbf{R}),\left(M_{p} g\right)_{p>0}$ are approximate units bounded in $S^{1}(\mathbf{R})$ (cf. [7, Chapter 1, $\left.\S 6.4,(8)\right]$ ). Therefore, we get $S^{1}(R)=L^{1}(R)[8$, p. 42]

It should be mentioned that for $G=Q_{p} \times{ }_{s} Z, S^{1}(G / Z)=L^{1}(G / Z)$ and $S^{1}\left(G / Q_{p}\right)=L^{1}\left(G / Q_{p}\right)$ for every Segal module.

Proposition 2. Let $H$ be an open subgroup of $G$. Let $S^{1}(H)$ be a Segal algebra on $H .\left(x_{\alpha}\right)_{\alpha \in A}$ denotes a complete system of representatives of $G \bmod H$. For $\alpha \in A$ let $f_{\alpha} \in L^{1}(H)$ be defined by $f_{\alpha}=\left.\left(L x_{\alpha}^{-1} f\right)\right|_{H}$.

$$
S^{1}(G)=\left\{f \mid f \in L^{1}(G), f_{\alpha} \in S^{1}(H), \alpha \in A \text {, and } \sum_{\alpha \in A}\left\|f_{\alpha}\right\|_{S^{\prime}(H)}<\infty\right\}
$$

is a Segal algebra with the norm $\|f\|_{S}=\Sigma_{\alpha \in A}\left\|f_{\alpha}\right\|_{S^{\prime}(H)}$.

Proof. A routine computation shows that $\left(S_{1}\right)$ and $\left(S_{2}\right)$ are fulfilled. Since for $f \in S^{1}(G), y \in G, \alpha \in A$, we have

$$
\left(L_{f} f\right)_{\alpha}=L_{\xi} f_{\beta} \text { with } y^{-1} x_{\alpha}=x_{\beta} \xi^{-1}(\beta \in A, \xi \in H),
$$

it follows that $S^{1}(G)$ is left invariant and $\left\|L_{y} f\right\|_{S}=\|f\|_{S}$. For $f \in S^{1}(G)$,

\footnotetext{
'This example is due to H. G. Feichtinger (A characterization of Wiener's algebra on locally
} compact groups, Arch. Math. 29 (1977), 136-140). 
$y \in G, \varepsilon>0$ there exists a finite subset $F \subset A$ with

$$
\left\|L_{y} f-f\right\|_{S}<\sum_{\alpha \in F}\left\|\left(L_{y} f\right)_{\alpha}-f_{\alpha}\right\|_{S^{\prime}(H)}+\varepsilon / 2 .
$$

There is a neighbourhood $V \subset H$ with

$$
\sum_{\alpha \in F}\left\|L_{y} f_{\alpha}-f_{\alpha}\right\|_{S^{\prime}(H)}<\varepsilon / 2, \quad y \in V .
$$

Since $y \rightarrow x_{\alpha}^{-1} y^{-1} x_{\alpha}$ is continuous, we take a neighbourhood $U$ such that $x_{\alpha}^{-1} U^{-1} x_{\alpha} \subseteq V$. For $y \in U$ we obtain, by (7)-(9),

$$
\left\|L_{y} f-f\right\|_{s}<\varepsilon \text {. }
$$

Proposition 3. Let $G$ be a nondiscrete, Abelian group and $U$ an open, nontrivial subset of $G$. There exist a Segal algebra $S^{1}(G)$ and $f \in S^{1}(G)$ such that $f \cdot C_{U} \notin S^{1}(G)$.

Proof. If $G$ is connected, Proposition 3 is trivial (take $S^{1}(G)=L^{1} \cap$ $C^{0}(G)$ ). It suffices to prove Proposition 3 for the compact case (every locally compact Abelian group has an open subgroup of the form $\mathbf{R}^{n} \times K$, where $K$ is compact).

Case A. The support $\hat{S}$ of $\hat{C}_{U}$ is infinite. Define

$$
S^{1}(G)=\left\{f f \in L^{1}(G), \sum_{\hat{x} \in \hat{S}} \frac{1}{\left|\hat{C}_{U}(\hat{x})\right|}|\hat{f}(\hat{x})|<\infty\right\}
$$

and

$$
\|f\|_{S}=\|f\|_{1}+\sum_{\hat{x} \in \hat{S}} \frac{1}{\left|\hat{C}_{U}(\hat{x})\right|}|\hat{f}(x)|
$$

(compare [8, §5, (VII)]). Now take $f(x)=1, x \in G$.

Case B. The support $\hat{S}=\left\{\hat{y}_{m} \mid 1 \leqslant m \leqslant M\right\}$ of $\hat{C}_{U}$ is finite. Consider $\hat{S}_{1}=\hat{S} \backslash\{\hat{e}\}$. There exists an infinite set $\hat{A}=\left\{\hat{a}_{n} \mid n \geqslant 1\right\}$ such that $\hat{y}_{m} \hat{A}$, $0 \leqslant m \leqslant M\left(\hat{y}_{0}=\hat{e}\right)$, are mutually disjoint (construct successively finite sets $\hat{A}_{n} \subseteq \hat{A}_{n+1}$ such that $\hat{y}_{m} \hat{A}_{n}, 0 \leqslant m \leqslant M$, are mutually disjoint, and set $\left.\hat{A}=\bigcup_{n>1} \hat{A}_{n}\right)$. Let

$$
S^{1}(G)=\left\{g\left|g \in L^{1}(G), \sum_{n>1} n^{2}\right| \hat{g}\left(\hat{a}_{n}\right) \mid<\infty\right\}
$$

and

$$
\|g\|_{S}=\|g\|_{1}+\sum_{n>1} n^{2}\left|\hat{g}\left(\hat{a}_{n}\right)\right|, \quad g \in S^{1}(G) .
$$

Now take $f \in L^{1}(G)$ such that $\left.\hat{f}\right|_{\hat{A}}=0$ holds, but $\hat{f}\left(-\hat{y}_{m}+\hat{a}_{n}\right)=\overline{c_{m}} / 2^{n}$, $1 \leqslant m \leqslant M, n \geqslant 1$, where $c_{m}=\hat{C}_{U}\left(\hat{y}_{m}\right), 1 \leqslant m \leqslant M$. Since 


$$
\widehat{f \cdot C_{U}}(\hat{x})=\sum_{m=1}^{M} c_{m} \hat{f}\left(-\hat{y}_{m}+\hat{x}\right), \quad \hat{x} \in \hat{G},
$$

we have $f \cdot C_{U} \notin S^{1}(G)$.

Proposition 4. Let $G$ be Abelian and not discrete. For id $\neq \sigma \in$ Aut $G$ there always exists a Segal algebra $S^{1}(G)$, which is not invariant under $\sigma$, i.e. there is an $f \in S^{1}(G)$ with $S_{\sigma} \notin S^{1}(G)\left(S_{a} f(x)=f(\sigma x)\right)$.

Proof. Assume for the moment that there exists a discrete sequence $\left(\hat{a}_{n}\right)_{n>1}$ in $\hat{G}$ tending to infinity such that

$$
\hat{\sigma} \hat{a}_{n} \neq \hat{a}_{m}, \quad n, m \geqslant 1,
$$

holds, where $\hat{\sigma}$ denotes the continuous automorphism on $\hat{G}$ defined by $\langle x, \hat{\sigma} \hat{x}\rangle=\left\langle\sigma^{-1} x, x\right\rangle, x \in G, \hat{x} \in \hat{G}$. Then take

$$
S^{1}(G)=\left\{g\left|g \in L^{1}(G), \sum n^{2}\right| \hat{g}\left(\hat{a}_{n}\right) \mid<\infty\right\}
$$

with

$$
\|g\|_{S}=\|g\|_{1}+\sum n^{2}\left|\hat{g}\left(\hat{a}_{n}\right)\right| \text {. }
$$

Since $\left\{\hat{\sigma} \hat{a}_{n} \mid n \geqslant 1\right\}$ is discrete and $\left\{\hat{a}_{n} \mid n \geqslant 1\right\}$ is closed, we can find a neighbourhood $\hat{V}_{n}$ of $\hat{\sigma} \hat{a}_{n}, n \geqslant 1$, which does not contain elements $\hat{a}_{k}, k>1$, or elements $\hat{\sigma} \hat{a}_{k}, k \neq n, k \geqslant 1$ (see (10)). Since $L^{1}(G)$ is regular, there exist $f_{n} \in L^{1}(G)$ such that $\hat{f}_{n}\left(\hat{\sigma} \hat{a}_{n}\right)=1$, supp $\hat{f}_{n} \subset \hat{V}_{n},\|f\|_{1}<1$ hold for all $n>1$. Set $f=\Sigma n^{-2} f_{n}$. We obtain $\hat{f}\left(\hat{a}_{n}\right)=0, n \geqslant 1$, hence $f \in S^{1}(G)$, but $\widehat{S_{\sigma} f\left(\hat{a}_{n}\right)=}$ $n^{-2}, n>1$, hence $S_{a} f \notin S^{1}(G)$.

The construction of a sequence with the properties above can be easily given by induction (Since $\hat{G}$ is not compact, outside of every compact set there always exists an element $\hat{a}$ with $\hat{\sigma} \hat{a} \neq \hat{a}$.)

LEMMA 2. Let $G$ be a nondiscrete and non-Abelian group. If there exists a compact, open, Abelian, nonnormal subgroup $H$, then there exists a non-rightinvariant Segal algebra on $G$.

Proof. Take for $S^{1}(G)$ a Segal algebra of the form in Proposition 2. There exist an element $y \in G$ and $\alpha \in A$ such that $x_{\alpha} H y$ is not a left coset. Therefore, we can choose $\beta \in A$ with $x_{\alpha} H y \neq x_{\beta} H$. We set $x_{\beta} U=x_{\alpha} H y \cap$ $x_{\beta} H \neq \varnothing$. Now, we take $0 \neq f \in S^{1}(G)$ such that $f_{\gamma}=0, \gamma \neq \alpha, \gamma \in A$. We obtain $\left(R_{y} f\right)_{\beta}=f_{\alpha} C_{U}$. Proposition 3 proves the existence of $S^{1}(H)$ and $f_{\alpha} \in S^{1}(H)$ such that $f_{\alpha} \cdot C_{U} \notin S^{1}(H)$.

LEMMA 3. Let $H$ be a closed, nonopen, nonnormal subgroup of $G$ such that $G / H$ has an invariant measure. There exists a non-right-invariant Segal algebra on $G$. 
Proof. $S^{1}(G / H)=L^{1} \cap C^{\sigma^{*}}(G / H) \neq L^{1}(G / H)$ is a Segal module. By Proposition $1, S^{1}(G)=T_{H}^{-1}\left(L^{1} \cap C^{0}(G / H)\right)$ is a Segal algebra. We take $y \in G, h \in H$ and a neighbourhood $U$ of the identity such that

$$
U h \cap U y H y^{-1}=\varnothing
$$

( $H$ is not normal and, in particular, $G$ is regular!). There exists $f \in L^{1}(G)$ such that supp $f \subset U$ and $f \notin S^{1}(G)$. Take a neighbourhood $V \subset U$ and $\dot{f} \in L^{1}(G / H)$ with supp $\dot{f} \subset \pi_{H}(V)$ and $\dot{f} \notin S^{1}(G / H)$. We set

$$
f(x)=\left\{\begin{array}{l}
\dot{f} \circ \pi_{H}(x) k(x) / T_{H} k \circ \pi_{H}(x), \quad x \in V H, \\
0, \quad x \notin V H,
\end{array}\right.
$$

where $k \in K_{+}(G)$ with supp $k \subset U$ and $k(x)>0, x \in V$. Take $y$ and $h$ such that (10) is fulfilled. $R_{h} f-f$ belongs to $\operatorname{ker} T_{H}$ [7, Chapter 8, \$2.5]; in particular, $R_{h} f-f \in S^{1}(G)$. If $R_{h y} f \in S^{1}(G)$ or $R_{h} f \in S^{1}(G)$ holds, the proof is finished. On the other hand, if $R_{h y} f \notin S^{1}(G)$ and $R_{y} f \notin S^{1}(G)$ hold, it follows from (7) that we have

$$
\operatorname{supp} T_{H}\left(R_{h y} f\right) \cap \operatorname{supp} T_{H}\left(R_{y} f\right)=\varnothing .
$$

Therefore $R_{y}\left(R_{h} f-f\right)$ does not belong to $S^{1}(G)$.

LEMMA 4. Let $G$ be a unimodular group and $H$ a closed, nonnormal subgroup of $G$. There exists a non-right-invariant Segal algebra on $G$, if $G$ is not discrete.

Proof. $H$ being a union of monothetic groups, we may assume that $H$ is isomorphic to $\mathrm{Z}$ or is compact and Abelian [2, p. 85], in particular, $H$ is unimodular. If $H$ is not open, one can use Lemma 3 , since $G / H$ has an invariant measure (cf. [7, Chapter 8, §1.4]). If $H$ is open, $H$ must be compact and Abelian. Now, Lemma 2 yields the result.

LEMMA 5. Let $G$ be a nonunimodular group. There exists a closed, nonnormal subgroup in $D=\left\{x \mid x \in G, \Delta_{G}(x)=1\right\}$.

Proof. Assume that every subgroup contained in $D$ is normal. There exists an open Lie-projective subgroup $L=\operatorname{proj} \lim L / K_{\alpha}$. Since $K_{\alpha}$ are compact, consequently, $K_{\alpha} \subset D$, we have $G=$ proj $\lim G / K_{\alpha}$; in particular, it follows that there is a compact, normal subgroup $K$ such that $G / K$ is a nonunimodular Lie group. Therefore, we can restrict our attention to Lie groups. For $x \notin D, N=H_{x} D$ is a closed, normal subgroup of $G$, where $H_{x}$ denotes the closed subgroup generated by $x$. ( $N$ is normal, since $G / D$ is Abelian; $N$ is closed, since $N / D$ is isomorphic to Z.) From [7, Chapter 3, §3.6(4)], it follows that $N$ is not unimodular. We write $K_{\max }$ for the maximal compact subgroup of the connected component $D_{0}$ of the identity in $D$. For any symmetric, precompact neighbourhood $U \subset D_{0}, U K_{\max }$ is a precompact neighbourhood in $D$. For $y \in U \backslash K_{\max }$, we have $z^{-1} y z \in\left\{y^{-1}, y\right\}, z \in G$. $\left(H_{y}\right.$ is not 
compact. This implies $H_{y} \cong \mathbf{Z}$, but Aut $\mathbf{Z}$ consists only of two elements.) Therefore, $U K_{\max }$ is invariant under the inner automorphisms, which implies $N \in[I N]$, in particular, $N$ is unimodular, a contradiction.

Proof of Theorem 3.

Case A. Assume that every closed subgroup is normal. From [9] it follows that $G$ contains an open, normal, Abelian subgroup of index 2, which does not belong to the center of $G$. Therefore, there exists a nontrivial automorphism on $H$ induced by an inner automorphism of $G$; using Propositions 4 and 2, we obtain a Segal algebra $S^{1}(G)$ such that $L_{y} R_{y}-S^{1}(G) \neq$ $S^{1}(G)$ holds, where $y$ is not contained in the centralizer of $H$.

Case B. Assume that $G$ contains a nonnormal, closed subgroup $H$. We may assume that $G$ is not unimodular (see Lemma 4). By Lemma 5 we can choose $H$ contained in $D$, in particular, $G / H$ has an invariant measure (cf. [7, Chapter 8, \$1.4D. If $H$ is not open, use Lemma 3. If $H$ is open, we can argue as in Lemma 4.

ReMARKs. (i) In Case $\mathrm{A}$ of the proof above, we give a more explicit construction of a non-right-invariant Segal algebra in the following way. Since $G=\mathbf{H} \times A$ is the direct product of the quaternion group $\mathbf{H}$ with a nondiscrete Abelian group $A$ (cf. [9]), we consider $L^{1}(G)$ as $L^{1}(A)^{8}$, i.e. for $g \in L^{1}(G)$ we write $f=\left(f_{h}\right)_{h \in \mathbf{H}}$, where $f_{h}=\left.f\right|_{\{h\rangle \times A}, h \in \mathbf{H}$. Take any Segal algebra $S^{1}(A) \neq L^{1}(A)$ and define $S^{1}(G)$ by

$$
\begin{gathered}
f \in S^{1}(g) \text { iff } f_{j}+i f_{k}, f_{-j}+i f_{-k}, f_{-1}+i f_{1}, f_{1}+i f_{-1}, \\
f_{-1}+i f_{-1}, f_{1}+i f_{1}, f_{k}+i f_{-j}, f_{-k}+i f_{j} \text { belong to } S^{1}(A) .
\end{gathered}
$$

If we take $g \in S^{1}(G)$ such that $g_{-1}=-i g_{1}=-g_{1}=i g_{-1} \notin S^{1}(A)$, we obtain

$$
\left(R_{-j} g\right)_{j}+i\left(R_{-j} g\right)_{k}=g_{-1}+i g_{-1} \notin S^{1}(A) .
$$

(ii) Let $G=\mathbf{Z}\left(q^{k}\right) \times{ }_{s} \mathbf{Z}_{p}$ be the semidirect product of the cyclic group of order $q^{k}$ with the $p$-adic integers, where $p$ divides $q^{k-1}(q-1)$. Every nontrivial subgroup of $G$ is open (we cannot dispense with Lemma 2).

\section{BIBLIOGRAPHY}

1. S. Grosser and M. Moskowitz, Compactness conditions in topological groups, J. Reine Angew. Math. 246 (1971), 1-40.

2. E. Hewitt and K. A. Ross, Abstract harmonic analysis, Vol. I, Springer-Verlag, Berlin and New York, 1963.

3. B. E. Johnson, Some examples in harmonic analysis, Studia Math. 48 (1973), 181-188.

4. E. Kotzmann and H. Rindler, Central approximate units in a certain ideal of $L^{1}(G)$, Proc. Amer. Math. Soc. 57 (1976), 155-158.

5. J. Liukkonen and R. Mosak, Harmonic analysis and centers of group algebras, Trans. Amer. Math. Soc. 195 (1974), 147-163.

6. R. D. Mosak, Central functions in group algebras, Proc. Amer. Math. Soc. 29 (1971), 613-616. 
7. H. Reiter, Classical harmonic analysis and locally compact groups, Oxford Math. Monographs, Oxford Univ. Press, Oxford, 1968.

8.,$L^{1}$-algebras and Segal algebras, Lecture Notes in Math., vol. 231, Springer, Berlin and New York, 1971.

9. S. B. Strunkov, Topological Hamiltonian groups, Uspehi Mat. Nauk. 20 (1965), No. 6 (126), 157-161. (Russian).

10. A. Weil, L'intégration dans les groupes topologiques et ses applications, Hermann, Paris, 1951.

11. A. B. Willcox, Note on certain group algebras, Proc. Amer. Math. Soc. 7 (1956), 874-879.

Mathematisches Institut, Universitït Wien, Strudlhofgasse 4, A-1090 Wien, Austria 